A $R$ R T T I I C C U L L O O $\begin{array}{lllllllll}R & E & F & L & E & X & I & O & N\end{array}$

\title{
LAS UNIVERSIDADES PRIVADAS Y LA TRANSFORMACIÓN DE LA EDUCACIÓN SUPERIOR: el CASO de Puebla, MéXICO (2012-2015)
}

\author{
PRIVATE UNIVERSITIES AND THE TRANSFORMATION OF HIGHER \\ EDUCATION: THE CASE OF PUEBLA, MEXICO (2012-2015)
}

*Historiador de la universidad de Arizona, Master en Estudios de Norteamérica por la Universidad de las Américas Puebla y $\mathrm{PhD}$ por la mism Universidad en nisma ción $y$ Teorín de la Cultura. Docente en Instituto Universitario Boulanger (Mexico) E-mail: alfonsoa.gomezri@udlap.mx

\section{Por: Alfonso Gómez Rossi*}

Recibido: 6 de marzo de 2017 - Aprobado: 13 de junio de 2017

\section{RESUMEN}

Las políticas neoliberales que se instituyeron en México a partir de la década del 90 transformaron la relación de la ciudadanía mexicana con las diversas universidades públicas del país Azteca. Mientras que en las décadas anteriores el ingreso a la universidad pública había sido legislado como un derecho al que podían acceder todos los ciudadanos, las políticas neoliberales exigían que a partir de ese momento sólo entraran los candidatos que aprobaran el examen con buenas notas y tuvieran buenas calificaciones de la preparatoria o bachillerato de los que provenían. La decisión tuvo consecuencias inesperadas para jóvenes de la generación x y los millennials ya que al no poder acceder a la universidad pública recurrieron a universidades privadas, que en la cultura general fueron llamadas "Universidades Patito" por no ser consideradas buenas instituciones para la educación superior. El surgimiento de diversas universidades las obligó a competir entre ellas para poder atraer nuevos alumnos, a partir de programas de estudio innovadores, que al mismo tiempo fueran redituables. Irónicamente, las universidades públicas al ver el éxito que tenían estas carreras las incorporaron posteriormente a su matrícula, convirtiendo a las universidades privadas pequeñas en laboratorios experimentales de conocimiento.

Palabras clave: educación, México, neoliberalismo, universidad, "Universidad Patito".

\section{ABSTRACT}

The neoliberal policies that were instituted in Mexico from the 90's transformed the relationship of Mexican citizenship with the various public universities of the Aztec country. Whereas in the previous decades the entrance to the public university had been legislated like a right to which all the citizens could accede, the neoliberal policies demanded that from that moment only the candidates who passed the examination with good grades and had good high school or high school grades from those who came. The decision had unexpected consequences for young people of the generation $\mathrm{x}$ and millennials since, being unable to access the public university, they turned to private universities, which in general culture were called "Duck University" because they were not considered good institutions for higher education. The emergence of several universities forced them to compete with each other in order to attract new students, based on innovative study 
programs, which were also profitable at the same time. Ironically, public universities seeing the success of these careers subsequently incorporated them into their enrollment, turning small private universities into experimental knowledge laboratories

Keywords: education, for profit education, Mexico, neoliberalism, university.

\section{Introducción}

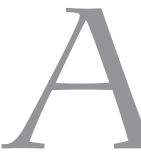

ño tras año se acumulan artículos en la prensa mexicana que señalan a una u otra institución de educación superior denominada universidad, instituto universitario o colegio como parte de un conglomerado nacional de "Universidades Patito." ${ }^{1}$ El nombre podrá sorprender a aquellos hispano-parlantes que se topan con un término que suena casi cómico; "universidad patito", ¿qué es una "universidad patito"? La respuesta, como se demostrará en líneas inferiores, no ha sido definida de manera satisfactoria, aunque en la opinión pública parece haber un consenso claro de lo que son. Las "Universidades Patito" (también conocidas como "universidades al vapor" o "de garaje") han sido definidas de varias maneras, desde instituciones que ofrecen licenciaturas y posgrados sin reconocimiento de la Secretaría de Educación Pública $(S E P)^{2}$ a universidades públicas que de acuerdo a las opiniones de ciertos personajes públicos merecen el apodo de "patito" (Rodríguez Gómez, 2004, p. 12).

A pesar de que ciertos sectores de la opinión pública afirman que estas instituciones de educación superior son una mala inversión para los estudiantes que acuden a ellas, este trabajo propone que las "Universidades Patito" o universidades con fines de lucro proporcionan a los alumnos que no pudieron ingresar a la universidad pública o privada más costosa, una educación a nivel licenciatura o maestría que los beneficiará en su futuro laboral. Estas universidades con fines de lucro apoyan a sus estudiantes en tres aspectos fundamentales: el primer motivo es económico, ya que por no tener el capital necesario para ingresar a las universidades particulares de más prestigio y no haber aprobado el examen de admisión es la opción que se adecua a las posibilidades económicas del estudiante y los familiares responsables por su manutención al momento de ingreso, ${ }^{3}$ en segundo lugar algunas de las instituciones de educación superior particulares ofrecen al alumno la oportunidad de cursar las licenciaturas en un período menor al que lo harían en alguna universidad pública o privada de renombre (tres años comparado con cuatro o cinco, que es el tiempo acostumbrado (http://ciudadanosenred.com.
1 Es sobre todo en las prensas locales donde se emplea el término de "Universidad Patito". Se puede buscar a través de google.com con las palabras 'universidades patito' con el nombre del estado junto $\mathrm{y}$ aparecen los siguientes resultados: en el caso de Puebla está como ejemplo: Denuncia UPAEP multiplicación de escuelas patito en gastronomía (http://www.e-consulta.com/), "Universidades sin acreditación se ofertan en la SEP" (http://www.e-consulta.com/), Sugiere Derbez dar un ultimátum a las universidades pato para elevar la calidad (http://www.econsulta.com/), "Pide Universidad Anáhuac acciones contra instituciones patito'" (http://www.econsulta.com/). En Coahuila "Alerta contra universidades 'patito' en Coahuila (http:/) www.vanguardia.com. $\mathrm{mx} /$ ), Clausurarán más escuelas 'patito'" (http://www.zocalo. com.mx/), "Señala UA de $C$ que universidades 'patito' saturan las profesiones (http:/) www.infonor.com. $\mathrm{mx} /$ ); en Tabasco "Se amparan las escuelas 'patito (sic.) (http:// www. tabascohoy. $\mathrm{com} /$ ), entre otros artículos de prensa. Roberto Rodríguez afirma que "en el período analizado [1993-2003] localizamos más de un centenar de textos de prensa, en distintos formatos y géneros que incluyen la denominación 'patito' en el encabezado principal, en los subtítulos, o bien, en su contenido" (12).

2 En este trabajo se utilizaran las siglas de la Secretaría de Educación Pública SEP cuando se mencione al organismo estatal.

3 Para obtener el costo de las carreras mejor catalogadas en ranking y hacer una comparación de sus precios y la duración ver: "Mejores universidades de México y sus costos" en ciudadanosenred.com.mx. 
4 El presente trabajo solo analiza licenciaturas innovadoras en las áreas de conocimiento económico administrativo, humanidades y ciencias sociales. mx) y el tercer motivo que es el que le interesa a este artículo es por qué las "Universidades Patito" en algunas instancias ofrecen carreras novedosas que rompen con el esquema disciplinar tradicional.

En algunas "Universidades Patito" se promueven licenciaturas o maestrías que salen del esquema tradicional del conocimiento, debido a que el ser instituciones orientadas a capitalizarse en la mayoría de los casos, deben buscar cómo atraer alumnos con planes de estudio innovadores que las diferencien de la competencia y al hacerlo logran generar los suficientes recursos económicos no solo para el sostenimiento de la institución, sino para generar una ganancia económica.

Las innovaciones educativas que se producen en estas instituciones de educación superior crean, mantienen o rescatan ciertas formas culturales del entorno local -como la gastronomía, artes plásticas o las artes circenses-, ${ }^{4}$ replanteando y redefiniendo la manera en la que se comprendían los métodos pedagógicos, los paradigmas educativos y obliga a replantear los esquemas educativos que tiene la SEP dándole a las "Universidades Patito" un rol importante dentro de la economía creativa de México. Este trabajo demostrará que las "universidades patito" generan un conocimiento subalterno que responde a las necesidades y realidades locales; al no derivarse de los modelos metropolitanos, suelen ser 'incomprendidos' por los estudios sobre producción/circulación del conocimiento y de educación superior.

\section{Análisis del término "Universidad Patito" en México}

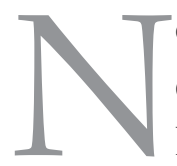

o existen obras que hablen favorablemente de la educación que se da en las instituciones de educación superior mexicana con fines de lucro; ninguna universidad se reconoce asimisma como "patito" y los dueños de las mismas tratan de hacer invisible la importancia que tiene para estas instituciones el generar los recursos suficientes no solo para sobrevivir, sino generar una ganancia mayor. Aunque en México no existe un trabajo que hable sobre los beneficios de las instituciones "patito," la autora estadounidense Anya Kamenetz en su libro DIY $U$, habla de una categoría de universidades norteamericanas cuyo fin también es generar capital a cambio de la impartición de educación superior: "Las universidades con fines de lucro han sido líderes en programas de aprendizaje interactivo y personalizado, innovación de programas en línea, evaluación basada en el desempeño [personalizado del alumno] y servicio al cliente basado en las necesidades [particulares] de los estudiantes. Tienen la ventaja de enfocarse 
exclusivamente en la impartición de conocimientos" (Kamenetz, 2010, p. 125). Se podría colocar a las "Universidades Patito" en la categoría descrita por Kamenetz, en el sentido de que son instituciones que lucran a partir de las colegiaturas de sus alumnos violentando con esta acción el espíritu moderno y moral del modelo kantiano-humboldtiano de la universidad pública en la edad moderna.

A Kamenetz le resulta chocante que una institución de educación superior tenga fines de lucro ${ }^{5}$ y que además sea un lugar donde no se lleve a cabo "investigación original que no tenga los fines inmediatos de generar ganancias" (Kamenetz, 2010: 127). A pesar de la crítica la autora propone que, por lo menos en Estados Unidos, la manera de pensar en la educación superior como un negocio es y ha sido tanto "necesario como una parte venerable del ecosistema educativo" (Kamenetz, 2010: 127). Es un hecho histórico que en Estados Unidos la educación superior siguió caminos distintos a aquellos trazados por Cambridge y Oxford y que fue alguien tan admirado como Benjamín Franklin quien las defendió alegando que el modelo que enseñaban era más práctico que la educación clásica de las universidades inglesas (Ruch, 2003 , p. 53). Las universidades con fines de lucro en Estados Unidos le prestan mayor atención a obtener resultados y disminuir los costos a diferencia de la típica universidad sin fines de lucro "sirviendo a un número similar de estudiantes" (Kamenetz, 2010: 126). El planteamiento de Kamenetz es el que rige e inspira el desarrollo de este capítulo ċlas universidades con fines de lucro o "patito" de la nación mexicana son laboratorios de innovación educativa? La respuesta se explorará en líneas posteriores, pero antes de proseguir es necesario preguntarnos icómo definiremos en el presente trabajo a las "Universidades Patito"? A continuación se hacen anotaciones sobre lo que implica definir a esas instituciones de educación superior mexicanas.

Por el artículo de Roberto Rodríguez "Entre lo público y lo privado: La polémica de las universidades patito en 2003" podemos inferir que las universidades que se analizan en este capítulo están definidas en el imaginario colectivo por la segunda palabra de su título: "patito" (Rodríguez, 2004: 11). El término "patito" es difícil de definir ya que es un vocablo que forma parte de la cultura mexicana y se refiere -en breve- a un producto de baja calidad: "El Diccionario Breve de Mexicanismos" define "marca patito" como "de marca poco conocida o mala" (Gómez de Silva, 2001, p. 134). ${ }^{6}$ Tomando en cuenta lo que es "patito" o "marca patito" podemos formarnos una idea de lo que es la "Universidad Patito," pero para complementar la definición anterior
5 Kamenetz escribe "La idea de que en el futuro las universidades con fines de lucro dominen el escenario puede parecer una pesadilla que daña la creencia de que la educación es un bien público. Yo he tenido esa reacción" (Kamenets 2010: 126).

6 El término "pato", aparte de definir al ave, se usa en México para describir a las conductas no honestas; Lo que se destaca de esta acepción es que coincidiría que el uso del término "marca patito" inició con la entrada de México en un esquema político-económico neoliberal, que es de gran importancia para este trabajo ya que se arguye en líneas inferiores que las "Universidades Patito" son también un reflejo del viraje de políticas estatales hacia el neoliberalismo económico. Rodríguez Gómez nos informa que "Parece que el término 'patito' comenzó a usarse a mediados de los años ochenta, en el mundo de los consumidores de cómputo... para establecer la diferencia entre equipos originales de marca, fabricados con componentes genuinos y cualquier variedad que careciera de estos atributos" (Rodríguez 2004: 11) 
se exploró como definen algunos mexicanos a las instituciones "patito" en algunos foros de internet. En yahoo respuestas se arrojan dos definiciones, la primera de el/la usuario/a Katiax que escribe:

Universidad "Patito" es una universidad privada la que, aunque cuente con el reconocimiento de validez oficial, es de baja calidad, tiene malas instalaciones y no enseña nada y/o hasta da buenas calificaciones a todos los alumnos sin esfuerzo y cobra de todo, casi hasta por respirar. Es importante saber que el reconocimiento de validez oficial NO garantiza que la universidad sea buena y es importante investigar el prestigio de la universidad privada antes de iniciar estudios universitarios. También es importante verificar todos los costos de la universidad para evitar sorpresas desagradables a la hora de graduarse (¿Qué es una universiad patito?, 2011).

Otra respuesta que contribuye a entender lo que significa para el público general la da Monik que define a la "Universidad Patito" como "una escuela o universidad "patito" es la que no está avalada por la SEP, mientras tenga el reconocimiento de validez oficial no hay problema" (patito?, 2011).

Las dos repuestas parecen indicar las concepciones que existen en el imaginario colectivo sobre el significado de "Universidades Patito". Entre otras contradicciones que mencionan ambas personas es que el reconocimiento de la SEP define si los programas son buenos o malos. Monik afirma que es suficiente el reconocimiento de la SEP para dejar de ser "patito", mientras que Katiax afirma que el aval de la Secretaría no es suficiente para darle calidad al programa universitario. José Armando Aguilar en su artículo "¿Educación o negocio? Más escuelas patito" da cinco lineamientos que él o la consumidor/a deben tener presente para determinar si una universidad es "patito":

1.- Son escuelas enfocadas hacia la carreras de mayor demanda, como derecho contaduría, administración, informática, ciencias de la comunicación, e ingenierías en computación e industrial.

2.- La gran mayoría [de los profesores] son contratados por hora clase. 3.- No llevan a cabo programas de investigación. De hecho la investigación requiere de inversiones que una escuela que busca únicamente el lucro no está dispuesta a realizar.

4.- Las áreas de estudio elegidas para impartirse son aquellas en la que es posible ofrecer una enseñanza de bajo costo, sin necesidad de 
equipos costosos.

5.- La institución cambia las carreras o los nombres de estas: si la demanda disminuye en una, es sustituida por otra carrera.

6.- En el sentido profundo del término, las universidades 'patito' no tienen ningún compromiso con el interés público o con la idea de servir a la sociedad. Las verdaderas universidades han enfatizado el servicio como una responsabilidad clave (Aguilar, 2006, p. 30).

Es de interés para este trabajo analizar los puntos que menciona el autor en las siguientes páginas; en una primera lectura es la definición más completa que hay a la fecha que define lo que son las "universidades patito," pero este trabajo cuestiona si algunos de los puntos que menciona el autor son tan negativos. El lineamiento 1, es indiscutible, la mayoría de las "universidades patito" ofrecen carreras que tienen una mayor demanda ya que son necesarias para la existencia de la institución de educación superior: Sin ellas, la universidad no sería un negocio redituable para el dueño y la existencia de la institución queda en entredicho.

Este es una de las cuestiones que, como señala Aguilar, demerita ante la opinión pública a las "patito" ya que se ha considerado que un afán de lucro por parte de los dueños es visto como un factor negativo, aunque criticar a las instituciones de educación superior particulares en México tiene décadas (Camp afirma que las críticas a la educación superior de carácter particular comenzaron a frenarse en la presidencia de Gustavo Díaz Ordaz, en la segunda mitad de la década del 60). El primer argumento de Aguilar enfatiza que las "universidades patito" tienen una finalidad exclusiva de lucrar, y que el afán de los dueños de aquellas instituciones de enriquecerse las convierte en instituciones educativas de baja calidad. Este argumento parece estar fundamentado en el artículo tercero de la Constitución mexicana que establece que "el Estado garantizará la calidad en la educación obligatoria de manera que los materiales y métodos educativos, la organización escolar, la infraestructura educativa y la idoneidad de los docentes y los directivos garanticen el máximo logro de aprendizaje de los educandos" (Artículo 3o) continuando en la segunda fracción

El criterio que orientará a esa educación se basará en los resultados del progreso científico, luchará contra la ignorancia y sus efectos, las servidumbres, los fanatismos y los prejuicios. Además: a) Será democrático, considerando a la democracia no solamente como una 
estructura jurídica y un régimen político, sino como un sistema de vida fundado en el constante mejoramiento económico, social y cultural del pueblo; b) Será nacional, en cuanto -sin hostilidades ni exclusivismos- atenderá a la comprensión de nuestros problemas, al aprovechamiento de nuestros recursos, a la defensa de nuestra independencia política, al aseguramiento de nuestra independencia económica y a la continuidad y acrecentamiento de nuestra cultura; c) Contribuirá a la mejor convivencia humana, a fin de fortalecer el aprecio y respeto por la diversidad cultural, la dignidad de la persona, la integridad de la familia, la convicción del interés general de la sociedad, los ideales de fraternidad e igualdad de derechos de todos, evitando los privilegios de razas, de religión, de grupos, de sexos o de individuos, y d) Será de calidad, con base en el mejoramiento constante y el máximo logro académico de los educandos (Artículo 3o).

Basado en lo que afirma el artículo tercero constitucional, Aguilar parece creer que enfocarse a carreras de mayor demanda le resta a las "Universidades Patito" y a sus alumnos de satisfacer algunas de las cláusulas del artículo tercero constitucional, argumento que no se sustenta a la lectura del mismo.

La noción de que una institución educativa tenga fines de lucro rompe con lo que Aguilar y una gran cantidad de mexicanos considera debe ser la función de la universidad moderna cuyos ideales fueron considerados durante gran parte del siglo XX como el de "ser un santuario en el que el conocimiento y la verdad pueden ser perseguidos -e impartidos- con impunidad, no importando cuan impopular, de mal gusto o políticamente heterodoxo el proceso pueda ser" (Hunter, 1991, p. 212) o en palabras de Enrique Cordero: "Liberar [la mente de los individuos] de prejuicios y errores que la cohibían; ensanchar sus límites, transformar caracteres, modernizar métodos y procedimientos... la enseñanza se difundió, se universalizó, dejó de ser de privilegio, ya que no se necesitaron fortuna personal o relaciones especiales para recibirla en forma selecta (Cordero, 1965, p. 173). Desde la perspectiva de Hunter y Cordero, las universidades con fines de lucro chocarían con esos ideales, porque son entendidas como empresas que generan ganancia a partir de la enseñanza de los ciudadanos mexicanos, que a la vez no están creando una ciudadanía libre en el aspecto ideal que se tuvo desde el liberalismo y la ilustración del siglo XVIII. Las "Universidades Patito" son comprendidas como negocios cuya finalidad es lucrar con el conocimiento que se le imparte a los estudiantes, lo que provoca críticas por parte de un 
gran número de intelectuales "que ejercen una influencia considerable en los medios de comunicación y la educación superior" (Camp, 1989, p. 40), al verlas no como vehículos de la libertad individual, sino como maquinarias que generan ganancias para sus dueños.

Hablar de las "Universidades Patito" como empresas que generan ganancias nos conduce a otro tema que trata sobre el trabajo que generan y el capital que generan. Durante la presidencia de Enrique Peña Nieto se ha presumido que la economía mexicana está en vías de mejoría en lo que toca al empleo gracias a la inversión extranjera directa que ha colocado en el país azteca 135 millones de dólares, traducido en la creación de 2.7 millones de empleos formales desde el 2012 (Lara, 2017). Esto desafortunadamente no representa un beneficio para todos los individuos mexicanos: los egresados de las carreras en ciencias de la salud, ciencias sociales, artes humanidades y educación perciben salarios menos favorables a otros egresados de acuerdo a la Secretaría de Trabajo y Previsión Social (¿A qué profesionales mexicanos subieron los salarios?, 2016). Algunas de las personas que laboran en esas áreas del conocimiento consiguen trabajo en las "Universidades Patito", pagando impuestos por el salario que detentan y las "Universidades Patito" pagan también impuestos por los siguientes derechos: Seguro Social, Afore, e Infonavit para algunos de sus empleados (Ley Federal de Trabajo, 2012), además de pagar el impuesto local del 3\% mensual (Impuestos sobre nómina 2017 , 2017) y los impuestos federales; además se pagan cuotas SEP anuales así como pagos por la titulación de los estudiantes.

Las "Universidades Patito" han sido responsables de brindar trabajos a muchos de aquellos licenciados o maestros que no pueden ingresar a dar clases a las universidades públicas por una serie de políticas particulares. ${ }^{7}$ En la mayoría de los casos que los profesores son contratados por hora clase lo que hace que la investigación sea poca o nula en la mayoría de los casos. ${ }^{8}$ Es una realidad que las instituciones "patito" no pueden sobrevivir de otra manera, ya que la institución debe generar los recursos económicos suficientes para su supervivencia así como para crear ganancias y esto a su vez obliga a la formación licenciaturas o maestrías novedosas para captar a los alumnos egresados de bachillerato. Una de las características interesantes de algunas universidades particulares es que para contratar a profesores, piden que tenga por lo menos dos años de experiencia, consideración que solo pueden cumplir cuando trabajan en las "Universidades Patito" antes de conseguir empleo en las universidades particulares de renombre.
7 De acuerdo a cifras que dio Guillermo Pablo López Andrade, director general de Acreditación, Incorporación y Revalidación de la SEP en "el primer trimestre [de] 2011] las universidades privadas aportaron 4.7 $\%$ del Producto Interno Bruto nacional, además de emplear a $373 \mathrm{mil}$ trabajadores-docentes y administrativos-en atención de 4.5 millones de alumnos en el país" (Alfaro http:// www.lajornadadeoriente.com.mx/).

8 Los trabajos pagados por hora clase generalmente implican que no se les pagará el Seguro Social. 
9 Entendido como un fenómeno social que se refiere a apoyarse en la creación de títulos universitarios, organizaciones profesionales y otras asociaciones como la manera de determinar la capacidad que tiene un individuo para realizar un trabajo (Credentialism).

10 Existe una ley federal sobre el servicio social que rige a la Ciudad de México. Cada entidad federativa de México elabora leyes sobre las que versa la obligatoriedad de realizar e servicio social.
Aparte del argumento economicista que se esgrime en las líneas anteriores, comenzamos este artículo escribiendo sobre los alumnos que escogen a las "Universidades Patito" porque no pudieron ingresar a otras universidades. Si la opción propuesta por los críticos fuera que mejor no estudiaran estos jóvenes, estaríamos esgrimiendo un argumento que se ciega a las realidades del mercado laboral. En las ciudades mexicanas se vive actualmente el fenómeno del credencialismo (Cerezo, 2016): ${ }^{9}$ mientras que tener una licenciatura no garantiza éxito económico, el contar solo con el diploma de bachillerato cierras las puertas al éxito económico futuro.

Para Aguilar el que no se genere investigación debilita a las "Universidades Patito", aunque existen universidades particulares afiliadas a universidades de renombre que tampoco realizan investigación. El lineamiento 4 de la definición de Aguilar es más difícil de probar ya que el autor no define que entiende por equipo costoso. Podemos suponer que se refiere a aparatos de tecnología avanzada que pueda ayudar a los alumnos de ciertas carreras a desarrollar tipos de conocimiento específico, aunque este argumento parece ir de la mano con el punto 1. Es importante destacar que el punto que se refiere al altruismo de la universidad y el impacto que esta puede tener en la sociedad la dejaría abierta a cuestionamiento, ya que las universidades o cualquier otro tipo de institución que oferte licenciaturas están obligadas por la SEP como requisito para la titulación de los alumnos a realizar el servicio social en dependencias gubernamentales, lo que implica que los conocimientos que realizan los estudiantes se aplican para el bienestar común de acuerdo a los lineamientos que impone el Estado. ${ }^{10}$

\section{Las garantías que deben ofrecer las “Universidades Patito”}

e ha problematizado en la sección anterior el significado que tienen en el imaginario colectivo las "Universidades Patito": aunque tienen 1 definiciones similares, no están unificadas de manera clara para la opinión pública. Para unos la "Universidad Patito" es aquella institución que carece del reconocimiento oficial de la Secretaría de Educación Pública, mientras que para otros puede contar con el reconocimiento de la SEP y al mismo tiempo ser considerada una "patito" por falta de instalaciones adecuadas, buen profesorado o un nombre apropiado. Este trabajo propone que las "Universidades Patito" mexicanas sean definidas como instituciones que si cuentan con el aval de la SEP, pero debido a una serie de factores no tienen un estatus alto dentro de la jerarquía universitaria nacional. 
El presente trabajo propone tres maneras distintas de determinar si una "Universidad Patito" es aceptable para que el público curse estudios en ellas o no. ${ }^{11}$ En primer lugar se propone que la universidad se encuentre registrada en la lista de universidades reconocidas por la UNESCO: si la institución no se encuentra dentro de esta lista se propone que esté acreditado por el sistema ISO 9001 y finalmente se propone que se haga una investigación profunda sobre el personal docente que labora en la institución que le interesa al estudiante.

Hablemos en primer lugar del reconocimiento de la UNESCO para determinar que una universidad es o no aceptable. Podrá parecer problemático el que un ciudadano mexicano busque a una institución no mexicana para comprobar que una universidad es "patito" o no, pero es necesario tomar en cuenta que uno de los problemas mayores en México es la percepción generalizada de que la corrupción que impera en el país azteca merma la calidad de las instituciones con aval del gobierno. El reconocimiento de la UNESCO conlleva lo que denominan en su página web una "garantía de calidad" que lleva implícito un control internacional (Education). Para las personas que pueden argüir que es innecesario obtener un reconocimiento de esa naturaleza, ya que las instituciones gubernamentales funcionan muy bien, se puede aclarar que México a través de la firma de varios tratados con la UNESCO reconoce la autoridad de este organismo, no como algo ajeno a la nación mexicana, sino como constitutivo de sus leyes nacionales. La UNESCO, a través de su organismo "Asociación Internacional de Universidades" 12 mantiene una lista de escuelas que denomina "La base de datos de Educación Superior del mundo" que "publica... el libro titulado International Handbook of Universities" (Ezell, 2005, p. 166). A la lista de universidades se puede acceder desde un servidor en cualquier parte del mundo y puede ayudar al futuro estudiante a decidir si la universidad que le interesa satisface los requisitos de la UNESCO.

Existen en la base de datos universidades que regionalmente se han considerado "patitos" en el pasado pero que cuentan con el aval de la UNESCO lo cual otorga cierto grado de legitimidad a la institución. ${ }^{13}$ El argumento de que es complicado buscar información por la web no parece tan convincente, sobre todo si se está buscando que el título y cédula profesional sean de una institución de educación que garantice legitimidad, aunque esta no pertenezca a las universidades privadas reconocidas como buenas instituciones y "permite darle cierta continuidad a las iniciativas planteadas por este organismo internacional" (Sagasti, 2011, p. 73).

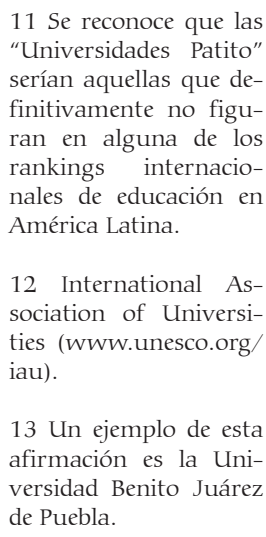

11 Se reconoce que las "Universidades Patito" serían aquellas que definitivamente no figuran en alguna de los rankings internacionales de educación en América Latina.

12 International Association of Universities (www.unesco.org) iau).

13 Un ejemplo de esta afirmación es la Universidad Benito Juárez de Puebla. 
14 ISO son las siglas de International Organization for Standardization. De acuerdo a Ezell se estableció para garantizar que las manufacturas internacionales fueran las mismas en cualquier parte del planeta. El ISO certifica que los productos que se hacen dentro de una compañía son de calidad (Ezell, 2005, p. 166).

15 El estado de Puebla ha obligado a las instituciones de educación superior a conseguir la certificación de algún organismo autorizado por la SEP (Cruz 2012).

16 Por ejemplo la ANUIES exige que se satisfagan criterio de investigación y difusión en el apartado IV.b), que como se escribió líneas arriba es una función que las "Universidades Patito" generalmente no practican.

17 Aunque estar sólo certificado por el ISO 9000 puede prestarse a algunos fraudes. En la década de los 80 una universidad en California trató de acreditarse solo con el ISO 9000 sin buscar la acreditación de otros organismos acreditadores legales (Ezell, 2005). La primera universidad acreditada que consiguió la certificación del ISO 9000 fue la Universidad del Este de Michigan en 1999, y a partir de entonces el reconocimiento de este organismo ha crecido en las universidades a nivel global. En México el estado de Puebla exige que para que las universidades no pierdan su RVOE se certifiquen por algún organismo aprobado por la SEP (Cruz, 2012).
La certificación por algún órgano acreditador entre las que se destaca el ISO 9001-2008 ${ }^{14}$ se ha convertido en ley en por lo menos una de las entidades federativas de la nación mexicana. ${ }^{15}$ El surgimiento de tantas instituciones que ofrecían licenciaturas y la percepción popular de que existía corrupción por parte de las autoridades correspondientes cuando se otorgaron los Reconocimientos de Validez Oficial (RVOE) fue determinante en la creación de legislación que exigía que las instituciones de educación superior fueran certificadas por algún órgano externo a la SEP. ANUIES (Asociación Nacional de Universidades e Instituciones de Educación Superior), FIMPES (Dederacion de Instituciones Mexicanas Particulares de Educacion Superior) y COPAES (Consejo para la Acreditación de la Educacion Superior), son tres ejemplos de organismos acreditadores que existen con la función de reconocer la calidad de los programas de estudio universitarios, aunque la realidad es que para la mayoría de las "Universidades Patito" los criterios de los tres organismos hacen que estas no sean acreditados por estos organismo ya que no satisfacen los criterios mínimos de pertenencia. ${ }^{16}$ Es entonces cuando la acreditación de otros organismos como el ISO 9000 se vuelve necesarios. Aclaremos que estar acreditado no significa que los programa académicos sean de calidad, lo que certifica es que la institución de educación superior en cuanto a lo administrativo tiene seriedad y el alumno puede confiar en que es una institución seria. ${ }^{17}$

Existe otra forma de comprobar si una institución "patito" es de mejor calidad o no, pero a diferencia de las dos formas anteriores esta es más complicada para el alumno ya que la información puede no encontrarse en internet y consiste en revisar la planta docente de la universidad en la que el alumno quiere ingresar. Si el profesorado es en un 100\% egresada de lo que hemos denominado en este trabajo como "Universidades Patito" o de la misma universidad en la que el estudiante desea ingresar, probablemente significa que la calidad de la educación no es tan buena como podría desearse. El autor de este texto está de acuerdo que puede haber un porcentaje de profesores egresados de "Universidades patito" pero que debe ser una minoría para garantizar que el nivel educativo sea bueno. En los casos mencionados es necesario recordar la expresión latina caveat emptor, comprendida como que el consumidor debe ser cauteloso. Revisar y verificar la información antes mencionada puede ser engorroso y pesado pero garantiza que el consumidor no será estafado ni perderá su tiempo y dinero en una institución que solo está interesada en lucrarse con el estudiante. 


\section{Las "Universidades Patito" como laboratorios experimentales: El efecto de las licenciaturas y maestrías de gastronomía}

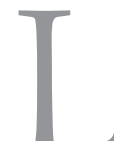

as dos décadas finales del siglo XX y la primera del nuevo milenio fueron testigos de la implementación del neoliberalismo, régimen Jeconómico que rompía con políticas que se habían instaurado desde el término de la Segunda Guerra Mundial (1945). El cambio de paradigmas económicos así como el desarrollo de nuevas tecnologías cambiaron el aspecto de la educación superior en México y el mundo. Tomando las palabras de Sagasti "el comienzo del siglo 21 es un periodo particularmente complejo y difícil en el cual la humanidad está experimentando cambios fundamentales en la forma dominante de generar conocimiento, en la base tecnológica, en la estructura de las actividades productivas y en las maneras en que estas corrientes se articulan entre sí, a través de los procesos de innovación" (Sagasti, 2011, p. 51). Las culturas en las que se desenvuelven los ciudadanos se han complejizado a partir de los cambios paradigmáticos que trajo consigo entre otros fenómenos, el neoliberalismo económico y los avances de la tecnología. Se propone en este apartado hacer un análisis somero de cómo cambió el modelo educativo en México durante las décadas de 1980 y 1990 a partir de las nuevas realidades económicas y sociales para posteriormente analizar el rol que tienen las "Universidades Patito" en un contexto geográfico específico, el de la ciudad mexicana de Puebla de Zaragoza.

Las instituciones de educación superior pública en México entraron en una crisis generalizada en las décadas del 70 y 80 que le dieron una mala imagen a la universidad pública frente a las clases medias altas y élites de México. Autores como Quezada, Avilés, Larroyo, Castañeda y Manjarrez en sus escritos sobre la sociedad mexicana y su relación con la educación escriben sobre esas décadas como aciagas para las universidades públicas. Jorge Castañeda sintetizó la naturaleza de la crisis en la universidad pública mexicana:

Gracias a la excelencia de la UNAM, desde los años veinte el país pudo contar con abogados, médicos, ingenieros y arquitectos de clase mundial con los cuales construir, administrarse y mantenerse saludable. Todo esto se modificó en los años setenta al dispararse la matrícula de educación superior, al tiempo que la educación primaria se estancaba en calidad y se inflaba cuantitativamente por la explosión demográfica de las décadas anteriores que... persistía. El gasto público en educación creció mucho pero igual la calidad se vio 
18 Aunque es necesario aclarar que existe la Secretaría de Educación Pública a nivel federal, y a nivel estatal resultando que cada entidad federativa tiene su propia Secretaría de Educación Pública. afectada y la cantidad de pesos gastados por cada alumno disminuyó. Luego después de las crisis recurrentes de 1982, 1987 y 1995, el gasto se recortó y se derrumbaron la calidad y el cupo en función de la demanda (Castañeda, 2011, p. 116).

El sistema de educación pública superior estaba en crisis y el Estado mexicano tomó la decisión de transformar las políticas educativas durante el sexenio de Carlos Salinas de Gortari (1988-1994). Siguiendo los ejemplos de Ronald Reagan en Estados Unidos y Margaret Thatcher en la Gran Bretaña en cuanto a la reforma del modelo económico mixto, se instituyeron en los tres países reformas que generarían cambios en lo que respecta a la educación superior. Interesa resaltar que los modelos de educación neoliberal que se establecieron a partir de la presidencia de Carlos Salinas de Gortari promovían una visión distinta al modelo educativo modernizante que existió durante casi todo el siglo XX. En el año 2000 el investigador Sergio Aguayo Quezada escribía sobre la transformación que se observaba en la educación y que tenía ya una década de antigüedad:

La reducción de los presupuestos oficiales, entre otros factores, ha provocado la caída en la calidad de la educación pública. Entre las consecuencias de dicha caída está la expansión de la educación privada que ha registrado un crecimiento espectacular pero que también tiene una calidad desigual (Aguayo Quezada, 2000, p. 90).

En las décadas del 80 y 90 las universidades que gozaban de mejor reputación eran las universidades privadas, como el Tecnológico de Monterrey y su sistema de filiales, la Universidad Anáhuac, la Universidad de las Américas entre otras. La percepción popular de que las universidades particulares ofrecían a los alumnos una mejor educación, aunada a criterios nuevos de admisión en las universidades públicas junto con el crecimiento de la educación superior que producía cada año mayor número de profesionales ayudó al incremento de universidades alternativas.

Es relevante destacar que la Secretaría de Educación Pública fue y ha sido responsable por implementar los nuevos paradigmas neoliberales encargándose de supervisar la implementación curricular de los diversos programas de licenciatura, maestría y doctorado a nivel nacional. ${ }^{18}$ Una característica particular de la SEP es que exige uniformidad en sus planes de estudio, como escriben Rich y Reyes: 
University curriculum in Mexico remains fairly standard regardless of whether private or public education is being discussed... Curriculum changes still have to be submitted to the government agencies... and whether one agrees or not with such conformity, at least on paper, the undergraduate studies do have much in common with those in Campeche or Cuernavaca (Rich, 2004, p. 1279).

La parte que sigue en el texto es interesante particularmente "esto incidentalmente no es necesariamente desastroso, porque a pesar de la crítica que provoca una supervisión central de que este sistema inhibe y limita la originalidad de la currícula, las alternativas caóticas también tienen sus desventajas" (Rich, 2004, p. 1279). Como explica Rich, el rol de la SEP es ambivalente. Por un lado el rol central que tiene, obliga a estandarizar a los programas educativos a nivel nacional, pero esta estandarización de programas presenta problemas en un entorno político, social y económico que es distinto al que se dio durante gran parte del siglo XX y obliga a cuestionar la manera en la que la SEP supervisa y organiza al sistema educativo nacional respecto a las necesidades de la sociedad actual.

Afortunadamente la SEP permite que, después de cumplir con los trámites correspondientes se otorguen RVOES para carreras nuevas que cumplan ciertos requisitos del organismo estatal o federal. Las "Universidades Patito" necesitan el reconocimiento de la SEP para ofrecer la carga académica y se ven en la necesidad de cumplir con las exigencias de la dependencia, pero al mismo tiempo necesitan generar tipos de conocimiento que las distingan de la competencia. La necesidad de capitalizarse las obliga a ofrecer las carreras de mayor demanda (ejemplos de carreras que poseen la mayoría de las universidades son: derecho, administración, contaduría, entre otros). En una sociedad en el que todas las universidades ofrecen las mismas carreras algunas de las instituciones desarrollan carreras que son novedosas y que funcionan como un laboratorio en el cual guían a otras instituciones para determinar si son o no redituables y convenientes las nuevas carreras. Un claro ejemplo de la afirmación anterior es la institución de la carrera de gastronomía en México.

Hasta la década del 80 estudiar una carrera asociada con el trabajo manual en la cocina de cualquier restaurante o incluso de la propia casa era un trabajo que se consideraba apto para el género femenino o para empleados subordinados que no tenían otra opción mas que trabajar en la cocina. En 
19 Es necesario aclarar que si había un interés por estudiar carreras que si involucraban a la gastronomía pero como parte de otra carrera: Así encontramos que la Licenciatura en Administración de Instituciones del ESDA (instituto que después se incorporaría a la Universidad Panamericana) ofrecía gastronomía como parte del plan de estudios, y la carrer se fundó desde 1967 aunque no comenzaron a impartir clases hast 1969 (http://www. up.edu.mx/document aspx?doc $=20823$ ). E centro de Estudios Superiores de San Ángel también ofrecía desde la década de los 70 una licenciatura en hotelería como parte del programa de estudios de hospitalidad (ver: http:// www.cessa.edu.mx quienesSomos.html).

20 Tatiana Gutiérrez afirma que la primera licenciatura en gastronomía fue la de la Universidad del Claustro de Sor Juana, pero se pone en duda la afirmación ya que parece más un afiche publicitario que una pieza seria (Gutiérrez, 2011)
México no existió un deseo por estudiar una carrera como gastronomía, ${ }^{19}$ hasta que ciertos segmentos de la población con mayor poder adquisitivo salieron del país a estudiar la carrera de gastronomía en Suiza en la década del 90. En los círculos sociales de clase media alta se vio con buenos ojos que sus vástagos fueran a Europa y tomaran cursos de cocina en Francia, Italia y España. Los dos primeros países, incidentalmente, tienen una concepción distinta del chef o profesional de la cocina ya que en distintas épocas de la historia el dirigente de una cocina fue considerado una especie de héroe por su profesionalismo. Desde los cocineros de la corte papal en los siglos XV y XVI hasta los chefs franceses de los siglos XIX y XX su profesión era valorada por la aristocracia. Ejemplos del reconocimiento a los profesionales de la cocina los descubrimos en los casos de Francois Vatel que laboró con Luis II, príncipe de Borbón - Conde en el siglo XVII o Antonin Carême cuya fama se debió en gran medida al talento de su producción así como el que servían a las testas coronadas de Europa (Iturriaga, pp. 166-167). Aunque era una carrera que debía su éxito a estar asociada con la aristocracia, los herederos de la nobleza no estudiaban las artes de la cocina, aunque si lo hacían las clases medias que veían a la carrera de cocinero como un vehículo de ascenso social; era atractiva por ser una carrera corta, generalmente una carrera técnica que se cursaba en un par de años en los que el egresado podía trabajar de inmediato.

En México los institutos que se especializaron en ofrecer carreras técnicas, cursos de cocina, o capacitación para el trabajo en alimentos y bebidas o repostería descubrieron en la década del 90 que existía una demanda en el mercado mexicano por parte de egresados de bachillerato de estudiar una carrera en las modalidades de técnico de nivel medio superior o superior o de licenciatura en gastronomía. Los mayores atractivos para los estudiantes eran la posibilidad de viajar a lugares distintos a los que vivían y a la vez ejercer una carrera que al ser relativamente desconocida implicaba una ignorancia de las materias de matemáticas y química que se cursarían, además se asumía como una carrera fácil (Gutiérrez, 2011). La primera escuela que estableció la carrera de gastronomía en México, de acuerdo a su página web fue "El Colegio Superior de Gastronomía," que transformó la carrera técnica en licenciatura en 1992 (Superior de Gastronomía, 2016). Un año después la universidad del Claustro de Sor Juana en la Ciudad de México fundó la licenciatura de gastronomía en su plantel. ${ }^{20}$ 
La carrera de gastronomía se expandió a otras partes de México donde fue siguiendo un patrón similar al de la Ciudad de México. Primero se abría una escuela desconocida para la mayoría de la población que atraía alumnos interesados en esa área, y el éxito que se observaba inspiraba a universidades de renombre a abrir su licenciatura en gastronomía o artes culinarias. Es de particular interés el caso del estado de Puebla por el caso exitoso que tuvo el Instituto Culinario de México y el Instituto Suizo así como por que las universidades particulares y públicas serias al observar el éxito de la carrera de gastronomía la implementaron en sus instituciones, demostrando que las "Universidades Patito" fueron en el caso particular de la gastronomía un laboratorio de carreras innovadoras. ${ }^{21}$ El éxito que tuvo la carrera de gastronomía, solo se puede entender en el contexto educativo y cultural de la ciudad de Puebla de Zaragoza.

Puebla es la entidad federativa donde existen más instituciones de educación superior después de la Ciudad de México. 22 "Anualmente egresan de las universidades poblanas 20 mil jóvenes... de una matrícula de 180 mil alumnos en las más de 190 universidades asentadas sólo en la capital del estado" (Puga Martínez, 2012). La otrora Puebla de los Ángeles se caracterizó desde la época colonial por ser una ciudad en donde las élites de diversas regiones mexicanas mandaban a estudiar a sus vástagos sino los enviaban a cursar estudios al extranjero o a la Ciudad de México. Citamos al historiador poblano Enrique Cordero y Torres:

Puebla conquistó desde el siglo XVII, la fama de tener buenos colegios, fama que ha conservado y acrecentado [el texto fue escrito en 1963]. En efecto, de la capital del virreinato eran enviados a la Angelópolis los hijos -niños y niñas- de los señores principales, a las escuelas particulares atendidos por religiosos. Después de la independencia, en pleno período de efervescencia política y perturbaciones bélicas, de todas partes de la República llegaban niños a las escuelas angelopolitanas... En los largos años del porfiriato, los hijos de los próceres que no eran enviados a París a estudiar recibían educación en la ciudad de Puebla; igual cosa sucedió después de la Revolución de 1910, continuando hasta la fecha (Cordero, 1965, p. 133).

Desde la supresión del Colegio de San Luis en 1861,23 la única institución de educación superior pública que existió en la entidad poblana fue el Colegio del Estado, que fue la antecesora de la actual Benemérita Universidad
21 Algunas universidades que implementaron la licenciatura en gastronomía en Puebla fueron la Benemérita Universidad Autónoma de Puebla, la Universidad Popular Autónoma de Puebla, la Universidad Iberoamericana Golfo-Centro (que ya cerró la carrera), entre otras.

22 Existen 2196 universidades en el país, de las que 300 corresponden a Puebla (ALFARO GALÁN, 2011).

23 El Colegio de San Luis era una institución de educación particular dirigida por la Orden de Predicadores (Dominicos) 
24 Se considera "patito" a aquellas instituciones que no figuran en el ranking de universidades o que no están afiliadas a alguna de las universidades de prestigio de la ciudad de México o Monterrey.
Autónoma de Puebla, institución que tuvo el monopolio de la educación superior en la entidad poblana hasta la década del 70 cuando aparecieron dos universidades particulares en la entidad: la Universidad Popular Autónoma de Puebla fundada en 1973 y la Universidad de las Américas establecida en Puebla en 1971 (Cruz Sosa, E. L.; García Castro, P. E. \& Gatica Barrientos, E., 2010, p. 406).

Dos lustros más tarde se habían establecido "cinco nuevas universidades privadas: La Universidad del Valle de Puebla, la Universidad Cuauhtémoc, La (sic) Universidad Iberoamericana [Golfo-Centro], el Instituto Tecnológico de Puebla, y la Universidad Realística" (Cruz Sosa, E. L.; García Castro, P. E. \& Gatica Barrientos, E., 2010, p. 406). Fue en la década del 90 y la primera década del siglo XXI cuando los centros educativos que ofrecían licenciaturas, maestrías y doctorados proliferaron en la entidad federativa y surgió el fenómeno bautizado por la prensa como "Universidades Patito." Para fines de la primera década del siglo XXI la entidad poblana, que en casi toda su historia no había tenido institución que se pudiera denominar universidad, contaba con más de doscientas treinta y ocho instituciones de educación superior (Cruz Sosa, E. L.; García Castro, P. E. \& Gatica Barrientos E., 2010, p. 406) Con las facultades -otorgada por el estado a través de la Secretaría de Educación Pública- de dar títulos de licenciado y maestro en la mayoría de los casos con la cédula profesional, la credencial que acredita la personalidad laboral de un estudiante. El hecho de que existan tantas universidades concentradas en un territorio con un número limitado de alumnos de candidatos a estudiar una licenciatura obliga a las instituciones de educación superior a modificar la oferta educativa con la finalidad de atraer a un público mayor. Aparte de ofrecer las licenciaturas de mayor demanda para el sostenimiento de las mismas, las "Universidades Patito" de Puebla están ofreciendo otras carreras que son innovadoras y que pueden ser aceptadas o no por las universidades privadas más grandes en el futuro. Se buscó a través de internet en los portales de cada universidad que no está en los rankings de las mejores universidades, dentro del estado de Puebla. ${ }^{24}$ La búsqueda de los programas innovadores que no se encuentran en las universidades de renombre son: 


\section{Universidad}

Colegio Libre de Estudios Interdisciplinarios

Instituto Suizo de Gastronomía

Universidad Mesoamericana campus Puebla

Universidad del Valle de Puebla

Instituto Tito Puente

\section{Licenciatura innovadora}

Licenciatura en Criminología, Criminalística y Técnicas Periciales

Licenciatura en Criminología y

Criminalística

Licenciatura en Psicología Criminalística

Maestría en Producción Panadera

Licenciatura en Artes Escénicas y

Circenses

Licenciatura en Producción y Animación

Licenciatura en Música Popular

Aún está por verse si estas carreras logran generar un interés grande en la población como lo fue gastronomía, y si esto provocará que otras instituciones copien o se inspiren por estos programas tomando como ejemplo los éxitos o fracasos de estas licenciaturas.

\section{Conclusión}

pesar de la mala reputación que tienen las "Universidades Patito"
la necesidad de generar recursos económicos suficientes para su
supervivencia los obliga a ofrecer a la par de las carreras tradicionales, otras que rompen con el esquema tradicional educativo. Las "Universidades Patito" fungen como un laboratorio en el que se puede experimentar con campos de conocimiento que las universidades más tradicionales no pueden o quieren por una serie de consideraciones que implican una decisión de Estado o una razón pecuniaria (Kamenetz, 2010, p. 127). Aún no se vislumbra cuál será el futuro de las "Universidades Patito". Como menciona Roderic A. Camp hasta la segunda mitad de la década del 60, las universidades particulares fueron sospechosas para el Estado mexicano. Solo hasta la presidencia de Díaz Ordaz se comenzó a vislumbrar un cambio favorable en la percepción general hacia las instituciones de educación superior particulares (Camp, 1989, p. 40). El tiempo puede transformar la percepción de las "Universidades Patito" que están siendo controladas con mayor efectividad a través del Estado para que beneficien a los estudiantes y egresados. 


\section{REFERENCIAS BIBLIOGRÁFICAS}

¿A qué profesionistas mexicanos subieron los salarios? (2016, November 4). Excelsior. Retrieved May 6, 2017, from http://www.nvinoticias.com/nota/5437/que-profesionistasmexicanos-subieron-los-salarios

Aguayo Quezada, S. A. (2000). El Almanaque mexicano: Un compendio exhaustivo sobre México en un lenguaje accesible y claro (Vol. 1). Ciudad de México, Distrito Federal: Editorial Grijalbo, S.A. de C.V. y Hechos Confiables S.A. de C.V.

Aguilar, J. A. (2006). ¿Educación o negocio? Más Universidades Patito. Revista del Consumidor (PROFECO). Retrieved May 6, 2017, from http://www.profeco.gob.mx/revista/publicaciones/ adelantos_07/2835\%20universidadesOKMM.pdf

Artículo 3o. (n.d.). Retrieved May 7, 2017, from http://www.ordenjuridico.gob.mx/Constitucion/articulos/3.pdf

Alfaro Galán, A. (2011, Septiembre 29). En 10 años creció 66 por ciento el número de universidades privadas; en Puebla, 300: SEP. La Jornada de Oriente. Retrieved May 8, 2017, from http:// www.lajornadadeoriente.com.mx/2011/09/29/puebla/edu108.php Camp, R. A. (1989). Entrepreneurs and Politics in Twentieth Century Mexico. Nueva York y Londres: Oxford University Press. (Camp, 1989)

Castañeda, J. G. (2011). Mañana o pasado: El misterio de los mexicanos. Ciudad de México, Distrito Federal: Santillana Ediciones Generales.

Cerezo, H. (2016, Agosto 10). El "pobresor" universitario. Milenio.com. Retrieved Mayo 7, 2017, from http://www.milenio.com/firmas/hector_cerezo/pobresor_universitario-maestros_universitarios-universidades_privadas_un_negocio_18_790301018.html

Cordero y Torres, E. (1965). Historia compendiada del estado de Puebla (2nd ed., Vol. III). Puebla, MX: Publicaciones del Grupo Literario "Bohemia Poblana".

Credentialism (n.d.). Retrieved May 7, 2017, from http://www.encyclopedia.com/social-sciencesand-law/sociology-and-social-reform/sociology-general-terms-and-concepts-116\#3045300482

Cruz, E. (2012, July 18). 12 universidades serán clausuradas por no acreditar calidad. Sexenio Puebla. Retrieved May 7, 2017, from http://www.sexenio.com.mx/puebla/articulo.php?id=11958

Cruz Sosa, E.; García Castro, P. E. \& Gatica Barrientos, L. (2010). La oferta educativa profesional y su impacto en el mercado laboral. EABR \& ETLC Conference Proceedings. Retrieved May 7, 2017, from http://www.google.com.mx/url?sa $=$ t\&rct $=j \& q=\& e s r c=s \& f r m=1 \&$ source $=$ web\&cd $=$ 3\&ved=OCEYQFjAC\&url=http\%3A\%2F\%2Fwww.cluteinstitute.com\%2Fproceedings\%2F2010_Dublin_ETLC_Articles\%2FArticle\%2520557.pdf\&ei=s3a9UMmLEcGZqAHdmIDACg\&usg=AFQjCNF bt3Kck7lROguECyfgQZIFmyOYy

Diccionario de Mexicanismos/Academia Mexicana de la Lengua (2010). Siglo XXI. Retrieved May 8, 2017, from http://www.google.com.mx/url?sa=t\&rct=j\&q=\&esrc=s\& 
frm $=1 \&$ source $=$ web\&cd $=1 \&$ ved $=0 C C 4 \mathrm{QFjAA} \& u r l=h t t p \% 3 \mathrm{~A} \% 2 \mathrm{~F} \% 2 \mathrm{Fwww}$.academia. org . $\mathrm{mx} \% 2 \mathrm{Fpdfs} \% 2 \mathrm{FDiccMexicanismos}$.pdf\&ei $=\mathrm{vGu}$ 9UMHdOIrJqQH0m4HQCg\&usg = AFQjCNGuu8 CTBKLvPOfKBo6pT-NwILn93w.

Education (n.d.). Retrieved May 7, 2017, from http://www.unesco.org/new/en/education/ themes/strengthening-education-systems/higher-education/quality-assurance/rankings-forum/ presentations/

Etimología de Pato Diccionario etimológico. <http://etimologias.dechile.net/?pato>.

Ezell, A. \& Bear, J. (2005). Degree Mills: The Billion-Dollar Industry that has Sold over a Million Fake Diplomas. Nueva York, NY: Prometheus Books.

Gómez de Silva, G. (2001). Diccionario Breve de Mexicanismos. Ciudad de México, Distrito Federal: Fondo de Cultura Económica.

Gutiérrez, T. (2011). Gastronomía: una moda que si sabe. El semanario sin límites. Retrieved May 7, 2017, from http://elsemanario.com.mx/revista_semanal/17_23_dic/5/index.html

Hunter, J. D. (1991). Culture Wars: The Struggle to Define America: Making Sense of the Battles Over the Family, Art, Education, Law and Politics. Nueva York, NY: Basic Books.

Impuestos sobre nómina 2017 (2017, January 4). Retrieved May 7, 2017, from http://losimpuestos.com.mx/impuesto-sobre-nominas/\#Impuesto-sobre-Nominas-enPuebla

Iturriaga, J. N. (n.d.). Gastronomía: Historia ilustrada de México. Ciudad de México, Distrito Federal: Penguin Random House.

Kamenetz, A. (2010). DIY U. Edupunks, Edupreneurs, and the Coming Transformation of Higher Education. White River Junction (Kamenetz, 2010), Vermont: Chelsea Green publishing.

Lara, C. (2017, April 10). Peña Nieto destaca crecimiento de empleos formales en México. El Sol de México. Retrieved Mayo 6, 2017, from https://www.elsoldemexico.com.mx/mexico/sociedadmex/637237-pena-nieto-realiza-hoy-gira-de-trabajo-por-el-estado-de-mexico

Ley Federal del Trabajo (2012, Noviembre 30). Retrieved May 7, 2017, from https://www. personal.unam.mx/dgpe/docs/leyFedTrabajo.pdf (Impuestos sobre nómina 2017, 2017)

Puga Martínez, J. (2012, Julio 3). Huyen estudiantes de la violencia en el norte del país; Puebla es su destino. La Jornada de Oriente. Retrieved May 8, 2017, from http://www.lajornadadeoriente. com.mx/noticia/puebla/huyen-estudiantes-de-la-violencia-en-el-norte-del-pais-puebla-es-sudestino-junghans_id_4539.html

Rich, P. J. \& De los Reyes, G. (2004). Teaching Canada in Mexico. American Behavioral Scientist, 47 (10), 1278-1284.

Rodríguez Gómez, R. (2004). Entre lo público y lo privado. La polémica de las "Universidades "Patito" en 2003. Retrieved May 6, 2017, from http://www.google.com.mx/url?sa=t\& 
$r c t=j \& q=\& e s r c=s \& f r m=1 \&$ source $=$ web\&cd $=4 \& v e d=0 C E U Q F j A D \& u r l=h t t p \% 3 \mathrm{~A} \% 2 \mathrm{~F} \% 2 \mathrm{FW}$ Ww.ses.unam.mx\%2Fintegrantes\%2Fuploadfile\%2Frrodriguez\%2FRR2005c.pdf\&ei=d-u 7UNoBM3uqAHnuIHoDw\&usg=AFQjCNGu-2g5waOSb3gwVXCyYinwepK2uA

Ruch, R. S. (2003). Higher Ed, Inc. The Rise of the For-Profit University. Baltimore, MD: Johns Hopkins University.

¿Qué es una universidad patito? (2011). Retrieved May 6, 2017, from https://mx.answers. yahoo.com/question/index?qid=20110911151731AAcISpA

Sagasti, F. (2011). Ciencia, tecnología e innovación: Políticas para América Latina. Ciudad de México, Distrito Federal: Fondo de Cultura Económica.

Superior de Gastronomía (2016). Retrieved May 7, 2017, from http://sg.edu.mx/portal/ 

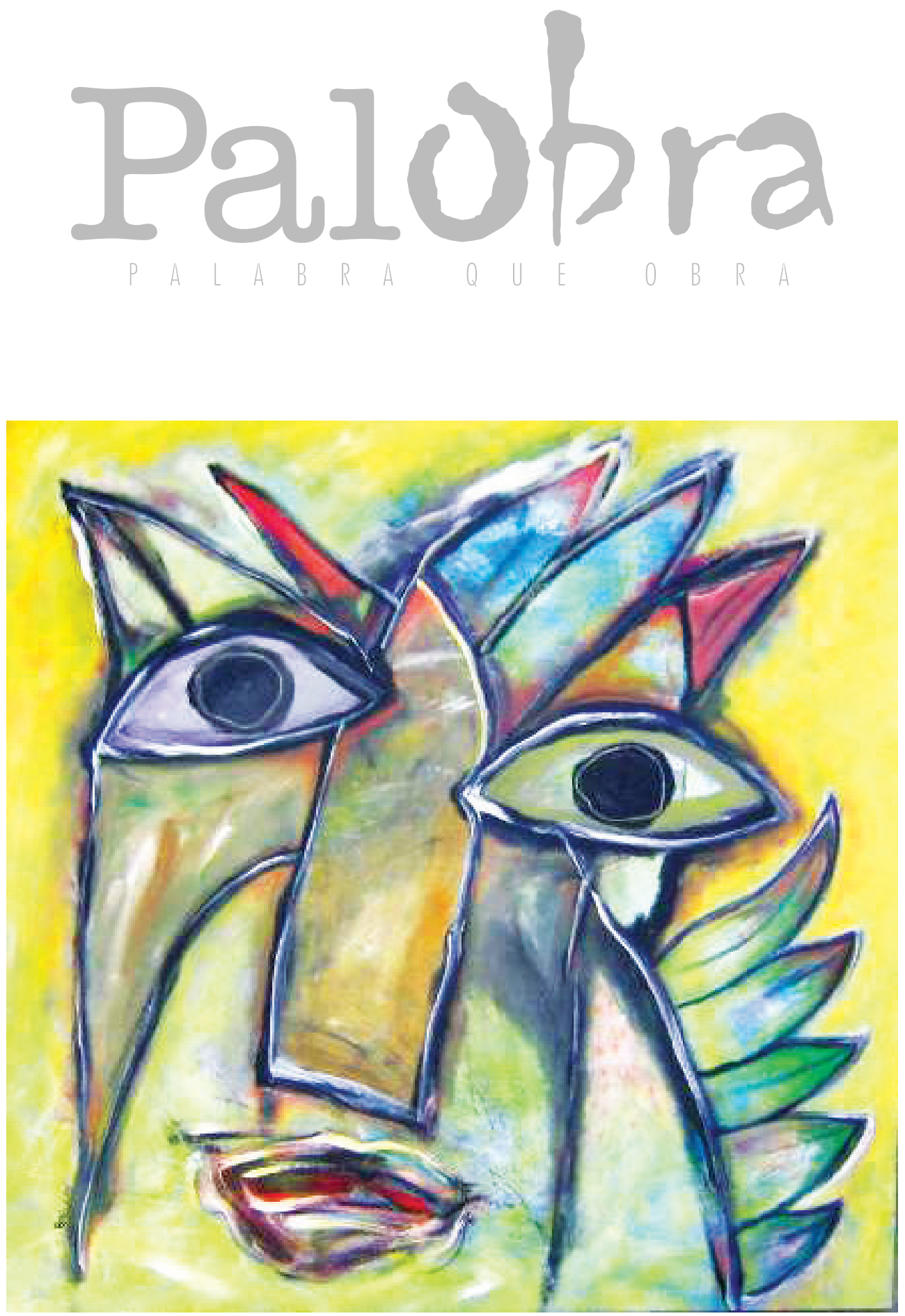

Obra pictórica de la serie "Rostros" de Eduardo Potrillé 